\title{
Observación, causalidad y explicación causal
}

\section{Fernando Cortés*}

Perfiles Latinoamericanos, 26(52) | 2018

DOI: $10.18504 / \mathrm{pl} 2652-001-2018$

Recibido: 15 de agosto de 2017

Aceptado: 30 de enero de 2018

\begin{abstract}
Resumen
En este ensayo se establece un diálogo entre las principales teorías epistemológicas sobre la causalidad que concluye con la diferenciación entre la causalidad y la explicación causal. Esta distinción es fundamental si se considera que la causalidad es un rasgo inaccesible de la naturaleza al que la ciencia se aproxima mediante explicaciones causales, es decir, basándose en modelos que organizan el material empírico y examinan el grado de correspondencia entre los resultados esperados, los proporcionados por el modelo y las observaciones. Los modelos correlacionales como las regresiones y los análisis de senderos, los modelos multinivel, el análisis de paneles y, en términos más generales, las ecuaciones lineales estructurales, entre otros, son concreciones del pensamiento conceptual de quien los diseńa (o escribe) y se someten al veredicto de la refutación. En consecuencia, la conclusión es que la causalidad no emerge de los datos ni es un resultado que surja de los modelos estadísticos.
\end{abstract}

\begin{abstract}
In this essay a dialogue is established between the main epistemological theories about causality that concludes with the differentiation between causality and causal explanation. This distinction is of great importance if one considers that causality is an inaccessible feature of the nature to which science approaches by means of causal explanations, that is, based on models that allow the organization of empirical material and examine the degree of correspondence between the expected results, provided by the model, and observations. As a particular case, correlation models such as regressions, trail analysis, multilevel models, panel analysis and, more generally, structural linear equations, among others, are concretions of the conceptual thinking of those who design them (or write) and submit to falsification. Consequently, the conclusion is that causality does not emerge from the data nor is it a result that emerges from the statistical models.
\end{abstract}

Palabras clave: causalidad, explicación causal, inferencia causal, modelos estadísticos, refutación, observación (método científico).

Keywords: Causality, causal explanation, causal inference, statistical models, refutation, observation (scientific method).

* Doctor en Ciencias Sociales, con especialidad en Antropología Social por el ciesas Occidente. Profesor Emérito, Flacso México/Pued,unam | fcortes@colmex.mx 


\section{Introducción: la causalidad en las ciencias sociales}

Un mundo compuesto de objetos permanentes constituye no solo un universo espacial, sino también un mundo dependiente de la causalidad, bajo la forma de relaciones entre las cosas como tales, y ordenado en el tiempo, sin continuas aniquilaciones y resurrecciones. Es, pues, un universo al mismo tiempo estable y exterior, relativamente distinto del mundo interior, y en el que el sujeto se sitúa como un término particular en medio de los demás. (Piaget, 1995: p. 11).

ए

ste trabajo examina, desde el ámbito de las ciencias sociales, cuán válido es inferir la causalidad a partir de datos observacionales. La preocupación surge de que hoy se asiste a la segunda oleada de trabajos que han emprendido este camino. Pareciera que la idea subyacente es buscar una vía alternativa para emular la investigación experimental, es decir, aquella que logra identificar el efecto de la variable experimental (C) controlando el papel de los factores que inciden en la relación entre la causa $\mathrm{C}$ y el efecto $\mathrm{E}$.

La primera oleada tuvo su origen en el trabajo de Lazarsfeld cuando este desarrolló el denominado modelo de análisis de covarianzas, ${ }^{1}$ el cual fue expuesto en 1946 en Cleveland, en un congreso de la Sociedad Americana de Sociología. Otro impulso fue el artículo de Simon publicado en 1957. Ambas contribuciones representan una clara continuidad de los aportes del genetista poblacional Wright (1934), quien por primera vez planteó la posibilidad de abordar el tema de la causalidad empleando técnicas estadísticas aplicadas a datos generados por observación.

Fundamentado en esos avances, Blalock (1964) cristalizó y desarrolló las ideas de los trabajos precedentes en un libro de título ilustrativo, Causal Inference in Nonexperimental Research. En este quedaron plasmados los esfuerzos de las ciencias sociales por inferir la causalidad a partir de observaciones obtenidas sobre todo, aunque no exclusivamente, por muestreo.

1 El análisis de covarianzas de Lazarsfeld fue la técnica más utilizada en América Latina en la década de 1960 para analizar la asociación entre tres variables dicotómicas; cuando el estudio involucraba a más se examinaba el cambio en la asociación entre X y Y ante las diferentes variables de control (t) tomadas de una a una, y solía concluirse, erróneamente, sobre dicha relación como si se hubiese controlado simultáneamente por el conjunto las mencionadas variables de control. 
Tales esfuerzos rebasaron las fronteras de los Estados Unidos. En Francia fue notable la contribución de Raymond Boudon (1967), quien publicó L'analyse mathematique des faits socieaux, cuyo tercer capítulo se dedicó al análisis de la causalidad a partir de datos no experimentales.

Sin embargo, al transcurrir la década de los setenta se debilitó el intento de inferir la causalidad con datos observacionales, un desaliento que aumentó en proporción directa a la incapacidad para controlar todos los factores relevantes que pueden influir sobre el vínculo causal, es decir, controlar a la manera de los experimentos en las ciencias naturales, o bien, ser capaces de aleatorizar sus efectos.

La segunda oleada de causalidad irrumpe en las ciencias sociales en la década de los noventa y toma pie, en primer lugar, en los trabajos de Holland (1986) en el plano filosófico. Este autor define el efecto causal como la diferencia entre el valor observado en la variable dependiente en el caso en que estuviese sometida a la variable causa y el efecto que se observaría si esto no fuera así. ${ }^{2} \mathrm{Y}$, en segundo lugar, en las propuestas de análisis estadístico de Rubin (1991) y Rosenbaum (2002).

En la actualidad las ciencias sociales siguen el camino abierto por Holland, en particular los derroteros señalados por King, Keohane \& Verba (2000), quienes sugieren que los valores observados de la variable dependiente están formados por la suma de un componente sistemático y otro aleatorio. El sistemático correspondería al valor esperado en la variable (esperanza matemática) medida en un conjunto de observaciones. ${ }^{3}$ En la práctica, las esperanzas matemáticas se estiman empleando técnicas estadísticas que operan sobre la matriz de varianzas y covarianzas de los datos.

En este ensayo se argumenta que los esfuerzos por destilar las relaciones de causalidad sobre la base de datos generados por observación parecen ignorar i) los planteamientos epistemológicos de Hume, ii) las discusiones que de ellos derivaron y que tuvieron un lugar destacado en las ciencias naturales en la vuelta del siglo XIx al xx (época en que nacieron la relatividad y la física cuántica), y iii) la distinción entre causalidad y explicación causal.

2 Esta definición de efecto causal tiene una estrecha correspondencia con el control experimental. Ciertamente, entrańa una situación en que, por un lado, se observa el efecto debido a la causa y, por otro, el valor que habría alcanzado si no hubiese estado sometida a ella. Este último caso es inobservable; es contrafactual y tiene dos características centrales: i) solo tiene validez conceptual en la medida en que no es posible retroceder el devenir, como si fuese una película en reversa, y ii) que en esta situación teórica estarían controlados los demás factores que pueden incidir en la relación causa-efecto y por tanto tendría la estructura del experimento.

3 Concebir la causalidad como la diferencia entre la esperanza matemática de los valores observados y de los que surgirían del contrafactual, da cabida a la construcción de modelos para estimar la esperanza matemática cuando la causa no hubiese operado. 


\section{El empirismo y la causalidad}

La revolución que experimentó la ciencia en los primeros años de siglo Xx puso en el tapete de la discusión algunos de sus conceptos básicos, entre estos la noción de causalidad. Los cuestionamientos tuvieron como punto de partida las ideas de David Hume en el siglo XviII, cuando este planteó el tema en un minucioso análisis de dos partes. En la primera concluía que la causalidad es un concepto que se origina en lo empírico y no en la razón. Al respecto sostenía que "ya que no es por el conocimiento o por un razonamiento científico por lo que derivamos la opinión de la necesidad de una causa para cada nueva producción, dicha opinión debe necesariamente surgir de la observación y experiencia" (Hume, 1992: p. 120). Y en la segunda, agregaba que era imposible fundar empíricamente el concepto de causalidad:

Como nuestros sentidos nos muestran dos cuerpos o cualidades, en ciertas relaciones de sucesión y continuidad nuestra memoria nos presenta solamente una multitud de casos en que hallamos siempre cuerpos, movimientos o propiedades análogas en análogas relaciones, de la repetición de una impresión pasada no surgirá una nueva idea original como la del enlace necesario, y el número de impresiones no tiene en este caso más efecto que limitarnos a una sola (Hume, 1992: p. 120).

Hume concluía que las relaciones necesarias en que descansa la causalidad no se pueden inferir de la experiencia sin importar el número de "impresiones". Para él era claro que no es posible derivar enunciados universales a partir de un cúmulo de enunciados particulares por numeroso que sea. De hecho, la imposibilidad de sustentar empíricamente los conceptos analíticos es uno de los problemas más recalcitrantes que ha debido enfrentar el empirismo.

Hume (1995: pp. 47-54) y los empiristas lógicos del siglo xx dividían los enunciados significativos en analíticos y sintéticos; ${ }^{4}$ y sostenían que las proposiciones formales son tautológicas y que las fácticas deben ser verificables. Asimismo, que cualquier proposición que no expresara nada que formalmente fuera verdadero o falso o no pudiera someterse a prueba empírica carecería de sentido (Ayer, 1981: p. 16), en tanto que los enunciados que no pertenecen a ninguna de tales categorías fueron calificados de metafísicos, por ejemplo, los relativos a la causalidad. ${ }^{5}$ Una cita de Hume ayuda a formarse una idea de la feroz crítica a la metafísica:

4 El valor de verdad de los enunciados analíticos se determina únicamente por los términos involucrados, en tanto que el valor de verdad de los enunciados sintéticos requiere la contrastación empírica.

5 Esta idea se retoma un poco más adelante al examinar las consecuencias de esta concepción sobre el abandono y el reemplazo de la idea de causalidad por la de relación funcional, en las ciencias naturales. 
Cuando persuadidos de estos principios recorremos las bibliotecas, ¡qué estragos deberíamos hacer! Tomemos en nuestra mano, por ejemplo, un volumen cualquiera de teología o de metafísica escolástica y preguntémonos: ¿Contiene algún razonamiento abstracto acerca de la cantidad y el número? ¿No? ¿Contiene algún razonamiento experimental acerca de los hechos y cosas existentes? ¿Tampoco? Pues entonces arrojémoslo a la hoguera, porque no puede contener otra cosa que sofismas y engaño (tomada de Enquiry Concerning Human Understanding, de Ayer, 1981: p. 115).

En 1950, Hempel publicó un artículo donde, manteniendo la posición básica del empirismo, modera la crítica a la metafísica excluyéndola de la posibilidad de generar conocimiento válido, pero reconociéndole la posibilidad de inspirar a la moral y tener atractivo emocional:

El principio fundamental del empirismo moderno es la idea de que todo conocimiento no analítico se basa en la experiencia. Llamemos a esta tesis el principio del empirismo. El empirismo lógico contemporáneo le ha añadido la máxima según la cual una oración constituye una afirmación cognoscitivamente significativa y puede, por lo tanto, decirse que es verdadera o falsa únicamente si es, bien 1) analítica o contradictoria, y 2) capaz por lo menos en principio, de ser confirmada por la experiencia. De acuerdo con este criterio, llamado criterio empirista de significado cognoscitivo, o de significatividad cognoscitiva, muchas de las formulaciones de la metafísica tradicional y grandes partes de la epistemología resultan carentes de significado cognoscitivo - independientemente de lo fructíferas que resultan algunas de ellas en sus connotaciones en virtud de su atractivo emocional o de la inspiración moral que ofrecen (Hempel, 1981: p. 115).

En los primeros años del siglo xx se fraguaban nuevas ideas que tomarían cuerpo a partir de la década del veinte en la reconstrucción de los fundamentos de la ciencia emprendida por el Círculo de Viena. De esas nuevas ideas, para los propósitos de este ensayo, es importante dejar constancia que destacados científicos impulsaron el abandono del concepto de causalidad y propusieron sustituirla por el de relación funcional.

Dicho planteamiento era del todo consistente con las ideas empiristas dominantes en la época. Si la causalidad debiera surgir, por ser un concepto sintético, de la observación y la experiencia, y nuestros sentidos son incapaces de mostrar las conexiones necesarias, entonces la causalidad podría catalogarse como metafísica y por tanto debiera ser repudiada.

En lo que sigue se aprovechará el estudio de Gil (1997) acerca del tránsito del empirismo desde la causalidad a la relación funcional, el cual realizó basándose 
en los escritos de algunos destacados científicos de entonces. Sobre Mach, dicho autor concluía: "Como ya había afirmado, la tarea de la ciencia consiste en dar cuenta de las relaciones funcionales de dependencia entre diversos elementos — sensaciones - de tal suerte que la noción de causa acusa ciertos vestigios de concepciones metafísicas que se eluden perfectamente bien si la sustituimos por la concepción matemática de función" (Gil, 1997: pp. 114-115). Y, al analizar la obra de Pierre Duhem, señalaba:

Las teorías físicas que pretenden ser explicativas de las apariencias sensibles están conformadas por dos partes radicalmente distintas: (a) una parte simplemente representa a la realidad y procura clasificar las leyes; (b) la otra —explicativapretende dar cuenta de la realidad que subyace a los fenómenos. Así divididas, Duhem considera que es falso que la primera depende de la segunda, el vínculo entre una y otra es frágil y artificial; la primera se ha desarrollado valiéndose del método adecuado de la teoría física, y la segunda es, sin más, un parásito de la primera (Gil, 1997: p. 120).

En la obra de Duhem se encuentran citas de Laplace, Ampàre, Fourier y otros renombrados científicos, además de Descartes, quienes rechazan la causalidad y abrazan en su lugar a las relaciones funcionales. Tal vez no sobra decir que Einstein estaba en desacuerdo con la idea de que la buena ciencia debería limitarse a registrar relaciones funcionales, pero esto es harina de otro costal (Holton, 1985: pp. 38-42).

\section{Causalidad y explicación causal}

Dentro del propio empirismo lógico surgieron algunas discrepancias que pusieron en duda sus principios. A partir del supuesto (que consideraban un hecho) de que las sensaciones daban el contenido fáctico a los enunciados, a ellas se debía su significado. Esta opinión se resumió en un lema: "el significado de una proposición consiste en su método de verificación” (Ayer, 1981: p. 18), lo cual no se pudo corroborar empíricamente.

Desde las filas del propio empirismo, Neurath (1932 a 1933) y Carnap (1932 a 1933) sostuvieron que si los enunciados elementales debían servir de fundamento a los intersubjetivos, ${ }^{6}$ ellos debían ser también intersubjetivos; tenían que referirse a acontecimientos físicos públicos. Desde ese mismo

6 Son los enunciados cuya verdad deriva del consenso entre los individuos. 
momento los enunciados elementales (o protocolares) ya no se consideraron incorregibles. $^{7}$

Russell Hanson avanzó en esta idea en su ensayo sobre la observación: "Pero la ciencia física no es solamente una sistemática exposición de los sentidos al mundo: también es una manera de pensar acerca del mundo, de formar concepciones. El observador paradigmático no es el hombre que ve y comunica lo que todos los observadores normales ven, sino el hombre que ve en objetos familiares lo que nadie ha visto anteriormente" (Hanson, 1989: p. 252). Bunge sintetiza con claridad la posición del empirismo lógico en nuestros días:

No tenemos acceso directo al mundo externo. Lo captamos solamente a través de la experiencia y la razón. Caeré en la tentación de la metáfora. La percepción y la acción median entre el mundo y nuestras ideas de él y nos dan la materia prima para la imaginación y el razonamiento. La elaboración resultante es un conjunto de ideas: imágenes, conceptos, proposiciones, diagramas, esquemas, clasificaciones, modelos y teorías. Verificamos estas ideas acerca de la realidad comparándolas con datos empíricos, no con el mundo mismo. En particular no confrontamos una proposición $p$ acerca de un hecho ${ }^{8}$ o hechos $f$ mismo, sino con algún dato (o datos) $e$ pertinente a $f$, es decir, alguna evidencia en favor o en contra de $p$. Podemos hacer esto porque $p$ y $e$ son proposiciones (Bunge, 1999a: p. 238).

Dada la imposibilidad del acceso directo al mundo externo, ¿`cómo seguir sosteniendo que el origen del conocimiento factual se encuentra en los hechos evitando la circularidad del argumento?, ¿cómo derivar la causalidad a partir de lo empírico, si este contiene conocimiento previo, incluidas las teorías?

En la sección anterior se ha visto que los científicos naturales de comienzos del siglo xx diferenciaban entre las leyes que rigen los fenómenos naturales y su explicación. Siguiendo esta misma línea de razonamiento se puede introducir una diferenciación entre suponer el comportamiento del mundo fáctico y explicarlo. Explicar es contestar a los porqué, "no nos contentamos con hallar hechos, sino que deseamos saber por qué ocurren” (Bunge, 1979: p. 561). Enseguida se exponen diferentes maneras de concebir la explicación causal.

Una de las posiciones asienta que "Dar una explicación causal de un acontecimiento quiere decir deducir un enunciado que lo describe a partir de las siguientes premisas deductivas: una o varias leyes universales y ciertos enunciados singulares — las condiciones iniciales_". (Popper, 1962: p. 57). En esta

\footnotetext{
7 Idea que pone en duda la validez de la teoría de la objetividad como reflejo de lo "real".

8 En este escrito, el término "hecho" se usa para referirse a sistemas, acontecimientos, procesos y fenómenos.
} 
concepción se distinguen dos partes: el explanandum y el explanans. El primero es "la oración que describe el fenómeno a explicar (y no el fenómeno mismo); el término explanans refiere a la clase de aquellas oraciones que se aducen para dilucidar el fenómeno" (Hempel, 1988: p. 249). Mientras que en el explanans se distinguen dos clases de oraciones: las que formulan las condiciones iniciales y las que representan las leyes generales.

En síntesis, esta manera de conceptuar la explicación causal reduce la causalidad al plano gnoseológico. En esencia se trata de subsumir enunciados particulares en oraciones que expresan leyes. ${ }^{10}$

La explicación por subsunción debe satisfacer las siguientes condiciones lógicas: i) que el explanandum sea una consecuencia lógica del explanans, ii) que el explanans contenga las leyes o regularidades necesarias para derivar el explanandum, y iii) que el explanans comporte un contenido empírico, esto es, que en principio debe ser comprobable por la observación o la experimentación a

9 Para Hempel, "El término ley sugiere la idea de que el enunciado en cuestión efectivamente ha sido confirmado por los elementos adecuados disponibles: como esta calificación en muchos casos es irrelevante para nuestros propósitos, emplearemos las expresiones 'hipótesis de forma universal' en lugar de 'ley general' y, si fuera necesario, estableceremos la condición de confirmación satisfactoria por separado" (Hempel, 1988: p. 233). La ley es conceptuada por Hempel como una hipótesis convalidada lo que concuerda con la posición de Bunge (1979: p. 335), aun cuando difieren respecto a si las leyes son solo producto del pensamiento o si "es una pauta estable que opera independientemente del conocimiento o de la voluntad humanos: es inherente a las cosas" (Bunge, 1999a: p. 47).

Debe notarse, asimismo, que si bien la aproximación de Hempel sistematiza el concepto desarrollado por Popper usa con frecuencia el término explicación y no explicación causal, excepto en la página 252 donde dice refiriéndose a la definición que "El tipo de explicación que hemos considerado hasta aquí comúnmente se denomina explicación causal” (Hempel, 1998: p. 252). Es probable que esta cautela se deba a que Karl Popper, después de rechazar la idea de explicación causal por ser tautológica o no refutable, propone concebirla como "una regla metodológica que se corresponde tan exactamente con el principio de causalidad, que éste podría considerarse como la versión metafísica de la primera. Se trata de la simple regla de que no abandonaremos la búsqueda de leyes universales y de un sistema teórico coherente, ni cesaremos en nuestros intentos de explicar causalmente todo tipo de acontecimientos que podamos describir: esta regla guía al investigador científico en su tarea" (Popper, 1962: pp. 57-60). Cuando Popper (1962: p. 137) dice que lo "guía" no significa que lo consiga de manera absoluta.

10 Para Hempel explicación y predicción comparten la misma estructura lógica lo que a menudo ha llevado a confundir ambos conceptos: "Se ha de señalar aquí que el mismo análisis, incluidas las cuatro condiciones necesarias, se aplica tanto a la predicción científica como a la explicación. La diferencia entre ambas es de carácter programático. Dado $\mathrm{E}$, es decir, si sabemos que el fenómeno descrito por $\mathrm{E}$ ha ocurrido y si se proporciona luego un conjunto adecuado de enunciados $C_{1}, C_{2}, \ldots \ldots ., C_{k} ; L_{1}, L_{2}$, ......., $\mathrm{L}_{\mathrm{r}}$, hablamos de una explicación del fenómeno que estudiamos. Si se proporcionan los últimos enunciados mencionados y se infiere $\mathrm{E}$ antes que suceda el fenómeno que se describe, hablamos de predicción" (Hempel, 1988: p. 251). Dada esta posición de Hempel cabe preguntarse (i) cómo consideraría la cientificidad de las disciplinas retrodictivas, o las que combinan predicción con retrodicción (ii) o cómo analizaría las explicaciones que recurren a la teoría del caos (determinístico) en que dados los enunciados y las condiciones iniciales es imposible determinar el resultado E. 
lo que se agrega la condición empírica de que las oraciones del explanans deben ser verdaderas (Hempel, 1988: pp. 249-250).

La manera como Hempel, en línea con Popper, entiende la explicación causal ha originado dos críticas: i) no sería exactamente una explicación sino más bien la subsunción de enunciados particulares bajo oraciones generales, y que ii) hace caso omiso de la parte ontológica de la causalidad y solo considera la parte lógica (Bunge, 1999b: p. 96). Este mismo autor interroga a manera de ejemplo para dar sustento a sus críticas: ¿cómo explicar las diferencias de los salarios pagados a hombres y mujeres con la misma preparación (estudios y experiencias similares) en el desempeño de igual trabajo? Si los hombres tienden a ganar en promedio 25\% más que las mujeres (regularidad que constituye el explanans), ¿puede ser esto una explicación de que Pedro gane 125 y María 100 (explanandum)? ${ }^{11}$ En las ciencias sociales esta regularidad suele plantear preguntas sobre los mecanismos que generan la discriminación de género. ${ }^{12}$ ¿Qué hay detrás de la diferencia?

Para responder esta pregunta Bunge propone el concepto de explicación mecanísmica que, por cierto, puede ser causal, estocástico o mixto, y se diferencia de la explicación a la Popper-Hempel presentada párrafos arriba en la cual la explicación científica debe hacer referencia explícita a los procesos, conocidos o supuestos, que vinculan la causa con los efectos (Bunge, 1999b: p. 98).

Además de esta diferencia entre las posiciones de Bunge y Popper-Hempel hay que asentar que la concepción de la causalidad en el primero deviene de una postura realista según la cual: "La causación no es una categoría de relación entre ideas sino una categoría de conexión constante y determinación que corresponde a un rasgo real del mundo fáctico (interno y externo), de modo que tiene índole ontológica, por más que como cualquier otra categoría de esa índole suscite problemas gnoseológicos" (Bunge, 1997: p. 21). Agrega, además, que asumirá explícitamente la posición de que el nexo causal o causación es

11 Este ejemplo se basa en el supuesto de que el explanans - expresado por la pauta regular "los hombres ganan 25\% más que las mujeres”, controlando por los demás factores que intervienen- es una ley o por lo menos una regularidad desde la cual se pueden extraer conclusiones. Se retoma así la idea de ley entendida como un enunciado que ha gozado de soporte empírico.

12 Bunge define mecanismo del siguiente modo: "Establezco que un mecanismo es un proceso en un sistema concreto, capaz de producir o impedir algún cambio en el sistema en su conjunto o en alguno de sus subsistemas. En resumen, un mecanismo es cualquier proceso que hace funcionar una cosa compleja. Dicho de otro modo, un mecanismo es el modo en que procede un proceso" (Bunge, 1999b: p. 55). Más adelante agrega las condiciones "para que una hipótesis o teoría mecanísmica sean tomadas en serio en la ciencia o la tecnología modernas, son que el mecanismo en cuestión sea concreto (en vez de inmaterial), sujeto a regularidades legales, (en vez de milagroso) y escrutable (en vez de oculto)" (Bunge, 1999b: p. 92). 
una cuestión ontológica, es decir, un rasgo propio del mundo fáctico (Bunge, 1997: pp. 18-22).

La concepción de Bunge es claramente opuesta a la de Popper-Hempel, para quienes la índole de la categoría de la causación es puramente gnoseológica; o sea, que la causación solo concierne a nuestra experiencia acerca de las cosas y a nuestro entendimiento de ellas sin ser un rasgo de las cosas mismas, por lo que toda referencia a la causación debe hacerse en lenguaje formal y no material (Bunge, 1997: p. 19).

Pareciera que no es un despropósito aceptar a la causalidad como categoría ontológica en las ciencias sociales. Dejando a un lado los argumentos filosóficos, suponer que algunos procesos sociales, entendidos como regularidades empíricas, se pueden explicar a través de mecanismos causales, proporciona un marco de referencia para entender fenómenos tales como las consecuencias de las intervenciones que realizan determinados actores como las agencias del Estado, las cuales están orientadas por el propósito de provocar cambios en la situación social de algunos sectores de la población.

Como síntesis parcial se puede señalar que el primero de los conceptos, el de Popper-Hempel, funda una relación de subsunción entre el explanans y el explanandum, mientras que la explicación macanísmica de Bunge echa mano de un mecanismo conocido o supuesto que estaría operando en el plano de la experiencia. Por eso este último concluye que "el llamado modelo de cobertura legal de la explicación científica es correcto pero incompleto, porque sólo tiene en cuenta la estructura lógica de la misma" (Bunge, 1999b: p. 100). ${ }^{13}$

A las dos formas de entender la explicación causal ya presentadas, hay que agregar la idea de explicación causal en la epistemología genética, idea que tiene sus propias peculiaridades. De inicio se instaura la distinción entre los planos

13 Cabe destacar que Bunge (1999b) señala una diferencia esencial entre la explicación en las ciencias naturales y aquella que se da en las sociales, lo cual rememora el argumento de Weber de que en estas últimas la sed causal es mayor que en las ciencias naturales. En estas, la explicación estaría compuesta por una teoría mecanísmica contrastable y circunstancias de las que se generaría el explanandum, mientras que en las ciencias sociales hay que agregar a la teoría mecanísmica contrastable con sus correspondientes condiciones, los valores y las normas, para obtener el explanandum. Enseguida un ejemplo de Bunge que sirve para entender mejor el concepto de explicación en ciencias sociales.

¿Por qué se estableció el Estado de bienestar?

Explicación posible:

Generalización: La pobreza es una fuente tanto de infelicidad individual como de desorden social. Dato: Hay personas pobres en nuestra sociedad.

Juicio de valor: La pobreza es indeseable, ya que es dolorosa y degradante para el pobre y peligrosa para el rico.

Norma: Para evitar las consecuencias del desorden social, elévese el nivel básico de vida mediante la redistribución de la riqueza a través de la recaudación de impuestos para sufragar programas sociales. 
empírico y teórico en el campo del conocimiento científico. ${ }^{14}$ Las sucesiones temporales de los eventos conducen por inducción a determinar leyes generales y descriptivas pero no explicativas. Se supone que las regularidades observadas son una manifestación exterior de las relaciones causales que existen en el mundo de los objetos (plano empírico) y que solo pueden reconstruirse por atribución a las relaciones empíricas, de las conexiones necesarias que se fijan en la teoría (García, 2000: pp. 204-207).

La idea de que la causalidad en la epistemología genética es de naturaleza claramente ontológica a diferencia de la explicación causal, así como la distinción entre los planos empírico y teórico, queda claramente fijada en el siguiente pasaje:

Explicar un fenómeno físico supone, ciertamente, el empleo de tales operaciones, pues la investigación de la causalidad siempre llega a superar lo observable y a recurrir a enlaces inferidos, y, en consecuencia, operatorios. Pero aquí se agregan, y esto es lo esencial, las respuestas del objeto, ya que hablar de causalidad supone que los objetos existen exteriormente a nosotros y que actúan los unos sobre los otros independientemente de nosotros: si el modelo causal adoptado comporta una parte inferencial es con el único propósito de alcanzar esas propiedades del objeto. (Piaget \& García, 1973: p. 7).

La diferencia esencial entre la explicación causal propuesta por la epistemología genética y la mecanísmica radica en la relación que vincularía el plano teórico con el de la experiencia. Según se ha visto, Bunge considera parcialmente verdadera la idea de explicación de Popper-Hempel porque se limita al plano de la deducción lógica, pero parece aceptar que la explicación requiere erigir las condiciones iniciales y el explanans para subsumir al explanandum. El concepto de explicación de Popper-Hempel usa la subsunción para vincular los enunciados teóricos con los de observación, pero en la medida que el concepto se enriquece con la inclusión de la experiencia sería necesario explicitar las relaciones de esta con aquellos.

Las investigaciones de Jean Piaget y Rolando García, basadas en un cúmulo importante de experimentos en psicología genética, señalan una diferencia neta entre los casos en los que el sujeto aplica sus construcciones operatorias - como las operaciones de clasificar objetos, ordenarlos, numerarlos, fijar seriaciones, regularidades (por ejemplo, las de las leyes empíricas), etc., donde los objetos se dejan tratar- de aquellos en los que los sujetos les atribuyen relaciones necesarias:

14 Hay que precisar que los estudios en psicología genética mostraron que la acción del niño es el origen común de los componentes analíticos y sintéticos del conocimiento humano. 
Cuando por el contrario una composición operatoria es atribuida al objeto, como la transitividad en el caso de la transmisión, cuando son los objetos quienes actúan, es decir, en este caso aseguran por sí mismos la transmisión, entonces es el sujeto el que se somete a los hechos. Queda claro que él conserva su propia actividad, o sea, que una operación atribuida es siempre, simultáneamente aplicada, aplicada y atribuida al objeto, pero la recíproca no es verdad puesto que una operación puede ser aplicada a los objetos sin serles atribuida: por ejemplo, diez guijarros no son diez a no ser que un sujeto los numere por su correspondencia con otros conjuntos, en tanto que un movimiento se transmite sin la intervención del sujeto —atribución- (Piaget \& García, 1973: p. 39).

El concepto de explicación desarrollado por la escuela ginebrina no solo concibe la causalidad como un fenómeno ontológico y distingue los planos teórico y empírico, sino que agrega que la explicación en general y la explicación causal en particular consisten en atribuir las relaciones necesarias a los objetos:

De este modo es posible dilucidar el pasaje del nivel en que se describe la regularidad —en el que afirmamos el "cómo" de la ley empírica — al nivel en el que tenemos razones para decir "por qué" es así. La base de la explicación causal, el fundamento de la atribución del "porqué", no puede venir de la experiencia directa ni de una potencia o categoría causal "innata" como acertadamente advirtió Hume: la base de una relación causal está en la teoría, y son las teorías las formas más desarrolladas de la construcción de sistemas de interpretación de los fenómenos (Piaget \& García, 1973: p. 41).

Y la atribución es posible porque ninguna construcción teórica con respecto a la realidad es ajena a la fuente original de todo proceso cognitivo: la interacción del sujeto con los objetos de su conocimiento (Gil, 1997: p. 207).

Sobre la base de estas discusiones resulta lícito preguntarse si del ajuste de una ecuación de regresión, de un análisis de trayectorias, de un sistema de ecuaciones lineales estructurales o, en términos más generales, de modelos estadísticos que buscan identificar estructuras de relaciones entre variables generadas por observación, es válido concluir que se ha develado la estructura causal que liga a los datos que representan los hechos. ${ }^{15}$

15 Se supone que las mediciones de las variables son válidas y confiables, es decir, que representan a los conceptos que se miden y que los errores de medición son tolerables o relativamente pequeńos. También se supone que los conceptos forman parte de sistemas teóricos dentro de los cuales se especifica su extensión, sentido y significado. 
Limitándose estrictamente a las ideas expuestas en esta sección la respuesta es enfáticamente negativa. En efecto, la ecuación o los sistemas de ecuaciones representan o pueden representar (no necesariamente representan) una explicación causal sugerida por el investigador que puede o no corresponder con los hechos. Esta explicación atribuye, probablemente con base en conocimiento científico y teorías, la causalidad a los observables. La atribución opera del plano del conocimiento al de lo empírico y no en sentido inverso. En esta perspectiva, los modelos estadísticos cuyos procedimientos de estimación se basan ya sea directa o indirectamente en la matriz de varianzas y covarianzas, cuando se emplean desde la óptica de obtener estimaciones "causales" se pueden concebir como formas de organizar el material empírico para someter a prueba las explicaciones causales representadas en dichos modelos y no como una manera de inferir las relaciones causales entre las variables (que se supone representan los hechos). Dependiendo de la bondad de ajuste y que se cumplan los supuestos en que descansan estos modelos, la conclusión lícita sería que la explicación causal propuesta por las ecuaciones es estadísticamente válida, pero no se debe olvidar que, de acuerdo con la teoría de la refutación de Popper, con base en este resultado no es pertinente derivar que la explicación propuesta es verdadera.

\section{La investigación experimental como modelo a seguir por la que se basa en la observación}

Con el propósito de facilitar la exposición de las ideas que se desarrollan en este apartado, se describen, en primer lugar, varios conceptos básicos que se refieren a los experimentos.

Un experimento consiste en provocar deliberadamente un cambio y observar e interpretar el resultado con una finalidad cognitiva (Bunge, 1979: p. 819). Añade a la observación científica el control, bajo supuestos teóricos, de un conjunto de factores que inciden o se supone que inciden sobre el resultado. En las situaciones experimentales el objeto está inmerso en un medio artificial que se encuentra bajo control en mayor o menor medida (Bunge, 1979: pp. 819-820).

La variable que manipula el experimentador se denomina variable estimulo o independiente $(\mathrm{X})$ y la que cambia, variable de resultado o dependiente $(\mathrm{Y})$. Para determinar las diferencias que provoca la manipulación de la variable independiente en la dependiente hace falta un testigo o un sistema de control. Este puede ser el sistema mismo cuando no se encuentra sometido a la operación de $\mathrm{X}$, siempre que sea estable en el tiempo. Si no se cumpliera este supuesto tendría que emplearse un sistema distinto aunque "equivalente", de modo que por la acción de la variable experimental aparecería una diferencia entre el sistema 
experimental y el de control. $Y$ en el caso de que haya variaciones individuales importantes, como ocurre en las ciencias sociales, hay que elegir conjuntos de individuos en lugar de casos individuales. Esto quiere decir que hay que elegir un grupo experimental y otro de control, pero esta selección debe ser de tal modo que la única diferencia entre ambos sea que uno recibe los estímulos del experimentador y el otro no (Bunge, 1979: pp. 830-831).

$\mathrm{Si}$ los resultados deben reflejar la operación de $\mathrm{X}$ y no del proceso de selección de las unidades observadas (es decir, en ausencia de sesgo de selección), los grupos experimental y de control tienen que ser homogéneos o relativamente similares en todos sus aspectos. Para lograr este propósito se pueden emparejar (aparear) las unidades garantizando su similitud en todas las variables observadas.

Sin embargo, esta no es la única técnica para lograr la homogeneidad de los grupos, la estadística pone a disposición de los investigadores los procedimientos de control de distribuciones y aleatorización. El primero consiste en igualar los grupos de tratamiento y de control en algunas medidas del colectivo, como promedios, medianas, varianzas, etc., olvidando las diferencias individuales, en tanto que el segundo consiste en formar ambos colectivos por aleatorización. Este procedimiento se basa en el principio estadístico de que si se toman dos muestras aleatorias independientes de una misma población ellas no presentarán diferencias significativas, es decir, que los grupos serán equivalentes u homogéneos. También se puede aplicar en situaciones experimentales en las que el investigador forma aleatoriamente, por ejemplo, dos grupos, $\mathrm{y}$ al azar se asigna el tratamiento a uno de ellos.

En los experimentos se satisfacen las tres condiciones de Mill, mismas que fueron replanteadas por Selltiz et al. (1959) para identificar relaciones causales. En efecto, el investigador manipula una variable C (causa) para obtener un resultado $\mathrm{E}$ (efecto), lo que garantiza la precedencia temporal, ${ }^{16}$ es decir, se observa $\mathrm{E}$ una vez que aconteció $\mathrm{C}$. Además, con la información que proporciona el experimento aplicación de $\mathrm{C}$ y cambio en $\mathrm{E}$ se puede establecer la relación entre $\mathrm{C}$ y E, es decir, que se puede dar cuenta del patrón de covariación que liga la causa con el efecto. Y, por último, los experimentos son diseñados de manera que se controlan los posibles efectos que puedan tener otras variables que representan otros hechos — sistemas, acontecimientos, procesos y fenómenossobre la relación causal.

16 Bunge (1997: pp. 65-69) afirma que, en la asimetría o sucesión causal, "El efecto aparece siempre que se hayan satisfecho las condiciones resumidas por $\mathrm{C}$, pero no necesariamente después de $\mathrm{C}$. Para emplear un término grato a los filósofos tradicionales, la causa es existencialmente previa al efecto; pero no tiene por qué precederlo en el tiempo". 
Hay que destacar que en los experimentos en las ciencias naturales se utilizan poderosas teorías, producto de siglos de trabajo acumulado, las cuales permiten identificar y "controlar"; en algunas oportunidades esto requiere del uso de medios complejos como aparatos e instrumentos especialmente construidos para controlar eficazmente los otros factores que tienen incidencia sobre la relación causa-efecto en estudio. Dependiendo de la naturaleza del problema se pueden lograr resultados equivalentes empleando la aleatorización estadística. En ambos casos el "control" debe entenderse como minimización de los efectos de los demás factores no explícitamente considerados y que intervienen sobre la relación. Nótese que se emplea "minimizar", pues difícilmente se podría sostener que dichos efectos se eliminan del todo.

En la sección introductoria de este ensayo se expuso que la segunda oleada de causalidad tomó pie en la idea de causalidad de Holland para quien el efecto causal sería la diferencia entre el valor observado en la variable dependiente si estuviese sometida a la variable causa y el efecto que se observaría si no lo estuviera.

La definición de causalidad de Holland —en la medida en que en una misma unidad se observa $Y$ bajo presencia y ausencia de $\mathrm{X}$ - satisface los requisitos de Mill (1952 [1875]): precedencia temporal de la causa sobre el efecto, relación entre $\mathrm{C}$ y E, y el control de las otras variables que podrían intervenir en la relación, pues los factores intervinientes estarían por igual manifiestos en presencia y ausencia de la causa $\mathrm{C}$.

Es evidente que la definición de Holland es en esencia teórica debido a que involucra una condición contrafactual; en cualquier situación real es imposible observar simultáneamente la variable $\mathrm{Y}$ sometida y no sometida a la variable X. El mismo autor concluye que en esta definición de causalidad hay una indeterminación básica que él denominó el problema fundamental de la inferencia causal: no se puede conocer con certeza un efecto causal (King et al., 2000: p. 90). A pesar de ello, la condición contrafactual involucrada en la definición señala el camino que debe seguir la investigación aplicada: la estrategia consiste en aproximarse lo más que se pueda a la situación ideal descrita por la definición de causalidad.

Para aproximar esta noción de causalidad a las condiciones en que operan las ciencias sociales, King et al. (2000: p. 93) la redefinen como "la diferencia entre el componente sistemático de las observaciones que se hacen cuando la variable explicativa tiene un valor y el componente sistemático de observaciones comparables cuando la variable explicativa tiene otro valor". Los componentes sistemáticos son los valores esperados en la variable (esperanzas matemáticas) medida en un conjunto de observaciones. En la práctica, las esperanzas matemáticas se estiman con técnicas estadísticas que operan sobre la matriz de varianzas y covarianzas de las observaciones. 
La posibilidad de que esta definición de causalidad arroje buenas estimaciones en la investigación empírica está condicionada, en el mejor de los casos, a determinar una situación que proporcione el contrafactual apropiado. Hay una vasta bibliografía ${ }^{17}$ dedicada a este tema que sobrepasa con mucho los alcances de este escrito, pero sí hay que tomar muy en cuenta la advertencia referida a los contrafactuales: "aunque se oponen evidentemente a los hechos, tienen que ser razonables; debería ser posible que el acontecimiento contrafáctico hubiera ocurrido en unas determinadas circunstancias" (King et al., 2000: p. 89).

En esta sección se ha visto que de las tres condiciones de Mill para determinar relaciones causales, la precedencia temporal y la asociación entre $\mathrm{C}$ y $\mathrm{E}$, en principio, no suscitan problemas mayores. El tercer componente, el control de los otros factores intervinientes se erige como un formidable obstáculo para identificar los vínculos causales.

Las ciencias de la naturaleza suelen diseñar experimentos basados en conocimientos que identifican cuáles son "los otros" factores que deben controlar y disponen o construyen artefactos que garantizan que su efecto sea mínimo, o bien, despreciable.

La otra vertiente para tratar el problema la proporciona el principio de aleatorización. En este caso no es necesario el uso de conocimiento teórico previo, excepto el estadístico. Esta disciplina señala que al formar aleatoriamente dos o más grupos, dependiendo del tamaño de la muestra, las variables intervinientes estarán por igual presentes en los diversos conjuntos y por lo tanto no intervendrán en la explicación de las diferencias.

En el experimento el control lo da la teoría sustantiva; en la aleatorización lo hace la teoría estadística.

Se ha visto que, desde el punto de vista teórico, la propuesta de Holland procura una solución al problema de determinación de la causalidad, equivalente al de la experimentación en sus dos vertientes - los experimentos propiamente como tales y los aleatorizados-. Sin embargo, como el concepto descansa en un contrafactual es imposible estimarlo con precisión a menos que se disponga de una situación idéntica al contrafactual. Por ello los estudios de causalidad realizados desde esta perspectiva se preocupan de elegir contrafactuales que fungen como grupos de control y que presenten diferencias pequeñas en el mayor número posible de variables observadas con el grupo "experimental"; no obstante, a diferencia de los experimentos en los que los grupos se construyen empleando procedimientos aleatorios, en este caso no se sabe qué papel juegan las variables no observadas, lo que se convierte en un fuerte obstáculo

17 Dos trabajos de consulta obligada para quienes decidan incursionar en este tema son el de Rubin (1991) y el de Rosenbaum (2002). 
para la identificación de la presencia de vínculos causales, así como para la estimación de su magnitud.

Tanto el experimento, así como la aproximación "contrafactual” inspirada en el trabajo de Holland, conceptuados desde una perspectiva estrecha parecieran centrarse en la experiencia como la forma privilegiada de vincularse con el mundo externo, opacando el papel del razonamiento. Sin embargo, al ampliar la mirada emerge una situación asaz distinta. En efecto, si se entiende la experimentación como una manera de formular preguntas al mundo en situaciones empíricas controladas (eliminando el efecto de todos los otros factores que pueden incidir sobre la relación causal) la respuesta que ofrece la "naturaleza" mostraría el vínculo causal que deriva de manipular X y observar el cambio en Y. Pero en esta manera de concebir el experimento queda en la penumbra el origen de la pregunta que llevó a conducirlo. En la gran mayoría de los casos la inquietud que guía la investigación toma pie en el conocimiento teórico y se sintetiza en una hipótesis que proporciona una explicación causal, pues le atribuye el sentido a la relación entre X y $\mathrm{Y}^{18}$

\section{Conclusiones}

A la vuelta del siglo xIx al xx, connotados científicos preocupados por el derrumbe de la teoría de Newton, se abocaron a trabajar sobre los fundamentos de la nueva ciencia que emergía en esos años. Se revisaron y discutieron los conceptos básicos tales como el espacio, el tiempo y, por supuesto, el de causalidad. Abrevaron de los trabajos de David Hume cuyos análisis le llevaron a concluir que la causalidad era un concepto sintético y que era imposible fundarlo empíricamente. La solución fue reemplazarlo por el de función. Para ellos hacer ciencia consistía en establecer regularidades y relaciones entre hechos.

Pero poco a poco fue emergiendo la diferenciación entre causalidad y explicación causal. Esta distinción es de suma importancia si se considera que la causalidad es un rasgo inaccesible de la naturaleza (recuérdese que no tenemos acceso directo al mundo externo. Lo captamos solo a través del razonamiento y la experiencia) al que se aproxima la ciencia mediante explicaciones causales, es decir, mediante modelos que permiten organizar el material empírico y examinar

18 A partir de estas ideas surge una serie de preguntas que exceden los estrechos límites de este texto. Por ejemplo, es natural inquirir por el origen de las preguntas que guían la investigación: ¿surgen del conocimiento acumulado y de las teorías previas?, ¿qué papel juega la observación?, ¿̨uál la creatividad y las vivencias del investigador? También afloran preguntas acerca de la acumulación del conocimiento logrado a través de los experimentos y en qué supuestos descansa respecto de las características del mundo externo y de su evolución. 
el grado de correspondencia entre los resultados esperados proporcionados por el modelo y las observaciones. En esta perspectiva, los modelos fundados en el conocimiento acumulado constituyen una explicación causal que será validada por su adecuación con las observaciones. Los modelos correlacionales como las regresiones, los análisis de senderos, los modelos multinivel, el análisis de paneles y, en términos más generales, las ecuaciones lineales estructurales, entre otros, son concreciones del pensamiento conceptual de quien los diseña (o escribe) y se someten al veredicto de la refutación. Una pizca de análisis de los "modelados" de otros investigadores o de los propios mostrará que ni la explicación causal ni la causalidad emergen de los modelos planteados.

La aproximación experimental, ya sea en la vertiente del experimento clásico o bien de los experimentos aleatorizados, es el camino que más acerca el conocimiento a detectar nexos causales. Sin embargo, la imposibilidad de anular, es decir, de hacer idénticamente iguales a cero los efectos de las "variables intervinientes" en los experimentos, o bien de garantizar la equivalencia absoluta de los grupos aleatorizados, impiden la certeza en la identificación de los vínculos causales. Como se ha visto, este mismo problema se presenta magnificado en los modelos correlacionales y en los casos en los que se intenta evaluar vínculos causales empleando contrafactuales.

En fin, pareciera que, hasta este momento del desarrollo de las ciencias, es imposible derivar causalidad a partir de las sensaciones como proponía David Hume y menos de las observaciones, según Holland. Pero de la mano de Popper, Hempel, Bunge y Piaget y García, la ciencia sí puede someter a prueba empírica explicaciones causales, que se tomarán como cada vez más verosímiles en la medida en que superen sucesivos contrastes empíricos (Stinchcombe, 1970: pp. 15-28).

\section{Referencias}

Ayer, A. J. (Comp.). (1981). El positivismo lógico. México: FCE.

Blalock, H. (1964). Causal Inference in non-Experimental Research. Chapel Hill: The University of North Carolina.

Boudon, R. (1967). L’anlyse mathématique des faits sociaux. París: Plon.

Bunge, M. (1999a). Buscando la filosofía en las ciencias sociales. México: Siglo XXI.

Bunge, M. (1999b). La relación entre la sociología y la filosofia. México: EDAF. 
Bunge, M. (1997). La causalidad: el principio de causalidad en la ciencia moderna. Buenos Aires: Sudamericana.

Bunge, M. (1979). La investigación científica: su estrategia y su filosofía. México: Ariel.

García, R. (2000). El conocimiento en construcción: De las formulaciones de Jean Piaget a la teoría de los sistemas complejos. Barcelona: Gedisa.

Gil, M. (1997). Conocimiento cientifico y acción social. Critica epistemológica a la concepción de ciencia en Max Weber. Barcelona: Gedisa.

Hempel, C. G. (1988). La explicación cientifica: estudio sobre la filosofía de la ciencia. Barcelona: Paidós.

Hempel, C. G. (1981). Problemas y cambios en el criterio empirista de significado. En Ayer, A. J. (Comp.). El positivismo lógico (pp. 115-135). México: FCE.

Holland, P. (1986, diciembre). Statistics and Causal Inference. Journal of the American Statistical Association, 81(396), pp. 945-960.

Hume, D. (1995). Investigación sobre el conocimiento humano. Madrid: Alianza.

Hume, D. (1992). Tratado de la naturaleza humana (1992), Tomo I. México: Gernika.

Holton, G. (1985). La imaginación cientifica. México: FCE.

King, G., Keohane, R. \& Verba, S. (2000). El diseño de la investigación social: la inferencia científica en los estudios cualitativos. Madrid: Alianza.

Lazarsfeld, P. (1974). La interpretación de las relaciones estadísticas como propiedad de investigación. En Boudon, R. \& Lazarsfeld, P. Metodología de las ciencias sociales II. Análisis empírico de la causalidad. Barcelona: Laia.

Mill, J. S. (1952 [1875]). A system of logic, Londres: Longmans.

Neurath, O. (1965). Proposiciones protocolares (1932-1933). En Ayer, A. J. (Comp.). El positivismo lógico (pp. 205-214). México: FCE.

Piaget, J. (1995). La construcción de lo real en el niño. México: Grijalbo.

Piaget, J. \& García, R. (1973). Las explicaciones causales. Barcelona: Barral.

Popper, K. (1962). La lógica de la investigación cientifica. Madrid: Tecnos.

F. Cortés | Observación, causalidad y explicación causal | Perfiles Latinoamericanos, 26(52) | FLAcso México DOI: $10.18504 / \mathrm{pl} 2652-001-2018$ 
Rosenbaum, P. (2002). Observational studies. Seg. edición. Nueva York: Springer Series in Statistics.

Rubin, D. (1991). Practical Implications of Modes of Statistical Inference for Causal Inference and the Critical Role of the assignment Mechanism. Biometrics, (47), 1213-1234.

Russell Hanson, N. (1989). Observación. En León, O. \& Pérez Ransanz, A. R. (Comps.). Filosofia de la ciencia: teoría y observación (pp. 216-252). México: Siglo XXI.

Selltiz, C., Jahoda, M., Deutsch, M. \& Cook, S. (1959). Research methods in social relations. USA: Henry Holt.

Simon, H. (1957). Models of man. New Cork: John Wiley.

Stinchcombe, A. (1970). La construcción de teorías sociales. Buenos Aires: Nueva Visión.

Wright, S. (1934). The Methods of Path Coefficients. The Annals of Mathematical Statistics, 5(3), pp. 161-215. 